\title{
¿ES POSIBLE UN MARCO JURÍDICO SUPRANACIONAL PARA EL Comercio Justo?
}

\author{
Alma Amalia González Cabañas
}

Resumen: Partiendo de la preocupación por proteger los intereses de consumidores, Organizaciones No Gubernamentales que coordinan el CJ y países miembros de la Unión Europea están inclinados a construir los cuadros jurídicos internacionales basados en la armonización de las normas para su regulación con el fin de reforzar su credibilidad y aumentar volúmenes de ventas. La experiencia de la cafeticultura sirve de ejemplo para mostrar las ventajas $y$ contradicciones de los marcos normativos en torno a los valores solidarios. Se analizan las gestiones del gobierno de Bélgica para promover una ley supranacional en la Unión Europea que reconozca al CJ; experiencia que muestra las vicisitudes planteadas.

Palabras clave: Comercio justo, mercados alternativos, agricultura orgánica, procesos de certificación.

Enviado a dictamen: 25 de noviembre de 2010 Aprobación: 16 de febrero de 2011

Revisiones: 2

Alma Amalia González, Doctorada en Estudios sobre América Latina, Investigadora Asociada "C" del PROIMMSE-IIA-UNAM, Temas de especialidad: Desarrollo Rural; y por El Colegio de la Frontera Sur (México), en Ecología y Desarrollo Sustentable (Tesis co-tutelar). Correo electrónico: agonzale@unam. mx; alma.amalia@gmail.com.
Abstract:Fair trade(FT), hasreached apoint ofgrowthunimaginable when it began. Departing from the wish to protect the interests of consumers, NGO that coordinate FT and certain European Union member countries, feel inclined to construct international legal frameworks basedon the harmonization of norms for itsregularization. The proposal claims to reinforce credibility in the system and increase the volume of sales. The experience of coffee growers serves as an example to demonstrate the advantages and contradictions of the normal conditions around solidarity values, certification processes and the demands of niche markets. Equally, the Belgian government's gestures to promote an international law in the European Union that recognizes FT are analyzed, an experience that demonstrates the difficulties that this creates.

Keywords: Fair trade, organic agriculture, alternative markets, certification process.

\section{Introducción}

$\mathrm{E}$ Comercio Justo ${ }^{1}$ (CJ) ha alcanzado un punto de crecimiento que nadie imaginó en los albores de Max Havelaar. "A través del apoyo creciente de los consumidores, CJ ha alcanzado una importante cuota de mercado a través de muchos productos en 20 países. En algunos mercados nacionales CJ representa entre 20 y $50 \%$ de la cuota de mercado en determinados 
productos", el número de organizaciones certificadas pasó de 508 en 2005 a 827 en 2009, con la participación de 60 países productores, y durante su último año de operaciones incrementaron en $11 \%$ el número de organizaciones certificadas, beneficiando a 1.2 millones de productores que comercializaron con este sello (FLO, 2009-2010).

Después de casi tres décadas de evolución del CJ, existen ventajas concretas para las organizaciones campesinas del sur que han participado en este dispositivo de coordinación (Raynolds, 2002). Este hecho es base fundamental del incremento en la aceptación de los consumidores. El reconocimiento y posicionamiento en ventas del CJ plantea, sin embargo, una paradoja: CJ aparece como una propuesta que enlazó los movimientos organizativos de productores en el sur con la movilización y concientización de consumidores del norte. El rol de las instancias del Estado y de la participación de la iniciativa privada fue por demás ajeno en ambos espacios norte-sur. Sin embargo, actualmente existe un creciente interés por el CJ de parte de estos agentes que inicialmente se mantuvieron distantes en la construcción del dispositivo de comercialización solidario.

El Comercio Justo constituye hoy por hoy un atractivo segmento de mercado donde otros agentes comerciales toman nuevas posiciones. Las empresas transnacionales dominantes en el sector cafetalero, pero en general del sistema agroalimentario, han retomado los postulados del CJ. Resultado de ello es la proliferación de diversas etiquetas que remiten a los postulados del CJ. Sin embargo, tales etiquetas carecen de los mecanismos de certificación y confianza de los dispositivos pioneros. Siendo que CJ nace de una iniciativa de movimientos sociales en forma de redes transnacionales, el papel del Estado quedó fuera del escenario. La ausencia de una instancia reguladora internacional ha llevado a la construcción de federaciones, como la International Social and Environmental Labelling and Accreditation Alliance (ISEAL) ${ }^{2}$ en diciembre de 1999 (Tallontine, 2001:
12). Sin embargo, el rol del Estado se hace cada día más urgente sobre todo por la presión que ejercen grupos de consumidores.

Partiendo de la preocupación de proteger los intereses de los consumidores en los países del norte las ONG que coordinan el CJ y algunos gobiernos miembros de la Unión Europea se muestran inclinados a construir marcos jurídicos internacionales basados en normas armonizadas. Se pretende que tales medidas puedan servir para reforzar la credibilidad en el sistema y ampliar los volúmenes de venta del CJ, ampliando con ello la participación de pequeños productores del sur que intervienen en el dispositivo de Comercio Justo.

Sin afán de negar las ventajas que tendrían eventualmente - la armonización y la legalización internacional del CJ, deseamos señalar algunos aspectos de riesgo a considerar. Para ello se hace una revisión de ciertas resoluciones, que tomadas desde órganos centrales de coordinación del CJ han tenido una influencia negativa para el fortalecimiento de la toma de decisiones de las organizaciones campesinas del sur. No obstante, el problema va mucho más a fondo, ya que la formalización del CJ implica ante todo enlazar los valores éticos a criterios y reglas que no son precisamente neutros. La participación del reino de Bélgica en la promoción de una iniciativa de ley a nivel europeo evidencia las contradicciones económicas que tal voluntad de solidaridad implica.

Este documento se basa en el desenvolvimiento de la cafeticultura campesina en el CJ, ya que como bien señala Fair Trade Labelling Organisations (FLO): "El café es un negocio millonario y sigue siendo uno de los productos agrícolas con más valor en el comercio mundial. Paradójicamente, para la mayoría de los 23 millones de productores de café, su cultivo supone mucho trabajo y pocos beneficios económicos" (FLO, 2011). Por otro lado, al remitirnos particularmente a la cafeticultura estamos analizando uno de los seis productos pioneros del cj labelizado que además se ha 
mantenido como líder por cuanto a volumen de ventas se refiere. El café es un ejemplo típico de producto de exportación introducido bajo el modelo productivo de grandes plantaciones en el siglo XIX. Sin embargo, la capacidad de innovación de los pequeños productores ha colocado a la vanguardia el sistema de manejo de las pequeñas unidades productivas, caracterizadas por su sustentabilidad ambiental a largo plazo (Moguel y Toledo, 1990; Soto, 2002). Siendo así, se reafirma la idea de que el CJ sólo toma cuerpo cuando se hace referencia a los valores locales - conocimientos, redes sociales y apropiación territorial - que definen la agricultura de pequeños productores.

Debe tenerse presente que, hasta el momento, se trata del único producto donde existe una norma -en el sistema Fair Trade Labeling Organizations (FLO) que mantiene fuera de participación de la certificación el café proveniente de grandes plantaciones de tipo privado. Este hecho representa sin duda un mayor grado de apropiación y participación en la toma de decisiones de las organizaciones campesinas del CJ, mostrando que uno de los aspectos centrales del CJ es hacer de él algo más que una opción de inserción al mercado, transformándolo en una estrategia de desarrollo local.

\section{Comercio Justo: zalternativa?}

Antes de adentrarnos en discusiones más detalladas sobre el desenvolvimiento de la iniciativa de CJ, vale la pena precisar qué es el precio justo y, sobre todo, cómo se expresa en la práctica. La definición del precio justo remite a remotas controversias sobre las concepciones de justicia y sobre las teorías que explican la construcción del precio. Si bien este documento no pretende profundizar en dichas cuestiones; sabemos por demás que es una discusión inacabada. Prueba de ello es, por ejemplo, la polémica que provoca CJ al haber fijado un precio mínimo que asegura cubrir los costos de producción, sin incorporar otro tipo de costos como los ambientales.
El precio mínimo se estableció a partir de un estudio de la Conferencia para las Naciones Unidas sobre Comercio y Desarrollo (UNCTAD). Posteriormente, fue añadida una prima de premio para el café orgánico. Actualmente, FLO (2011) declara que a las organizaciones de productores con certificado de CJ se les garantiza un precio mínimo de 125 centavos de dólar por libra para el café arábica lavado y 120 centavos de dólares de dólar para el café no lavado arábica, o el precio de mercado, en caso de que éste sea superior. Adicionalmente, CJ añade al precio de compra una prima de 10 centavos de dólar por libra, llamado premio social, mismo que debe ser utilizado por las organizaciones de productores en inversiones económicas y sociales en la comunidad y la organización.

Diversos autores han utilizado el término de mercados alternativos para referirse a aquellos dispositivos de comercialización que pretenden movilizarse al margen de las definiciones del mercado. La idea de CJ o comercio alternativo remite al reconocimiento de las desigualdades norte-sur, lo cual se expresa en el pago de un sobreprecio por los productos (Nigh, y Hernández, 1998; González, 2002; Raynolds, 2000; Taylor, 2005). Pero, ¿cómo se define el carácter alternativo del CJ? En este sentido, podríamos decir que la globalización se expresa en dos grandes tendencias comerciales: por un lado, la estrategia de la estandarización acompañada de una competitividad basada en la reducción de costos de producción. Sin embargo, una segunda expresión de la globalización es la creciente tendencia a la segmentación de la demanda. Es innegable que cada vez más los consumidores buscan opciones que les permitan reforzar sus diferencias identitarias. En este espíritu de construcción identitaria, una de las expresiones que cobra cuerpo es el interés por una relación "más justa" con los productores del sur. Así, Comercio Justo se plantea como una opción de diferenciación y como modalidad de comercialización de bienes no materiales. No obstante, el planteamiento de fondo del concepto "alternativo" remite a la 
posibilidad de incidir en la construcción del propio desarrollo de los grupos campesinos; a su capacidad de toma de decisiones para elegir el tipo de sociedad que ellos mismos construyen. En otros términos, habría que preguntarse si CJ es una opción alternativa de desarrollo que respeta las formas de vida de los pequeños productores del sur. O siendo más atrevidos en la pregunta, si no se trata simplemente de una modalidad de comercialización de los productos.

Diversos estudios sobre CJ ponen en evidencia las ventajas en términos comerciales que éste representa para los productores sureños. De igual manera señalan el dispositivo como un espacio de aprendizaje sobre los procesos de exportación y de relación institucional (Nigh, 1999; Raynolds, 2002). No obstante, la definición de reglas en el dispositivo de CJ introduce igualmente la pregunta ihasta qué punto los pequeños productores pueden participar en la definición de las condiciones de acceso a este nicho de mercado? Es decir; icómo se lleva a cabo la definición de los criterios que le dan un sentido concreto a los valores éticos que se incorporan? ¿Cuáles son las implicaciones de la movilización de los valores éticos en particular, de los procesos de exclusión social que resultan de la aplicación de estos valores? Realmente, la cuestión se plantea - en términos vastos - para todas aquellas opciones de diversificación de mercado en que han tenido oportunidad de participar los países del sur, tales como la agricultura orgánica, productos de la silvicultura con sellos verdes y aquellos que valoran los territorios y/o los conocimientos tradicionales.

\section{Escenario cafetalero}

De los productos que se comercializan bajo el sello de CJ, el café ocupa el primer lugar en volumen de ventas. El café ha sido durante el siglo pasado y hasta el presente el principal cultivo en términos financieros. Si bien es cierto que en todos los continentes el café se extendió en superficie a finales del siglo XIX, esto se hizo a partir del modelo de fincas o grandes plantaciones de propiedad privada. La consolidación de los Estados nacionales de países de trópico húmedo, particularmente en América Latina, hizo de la cafeticultura un eje rector de estrictas políticas de Estado que regularon el sector hasta los años 80 del siglo pasado. Este modelo fue igualmente seguido por países africanos. La incorporación de los países asiáticos al sector de la cafeticultura se produjo en tiempos más recientes - a finales del siglo pasadocomo estrategia productiva del Banco Mundial que habría de fomentar el desarrollo de países como Vietnam e Indonesia. Las presiones actuales por la liberalización de las economías ha traído consigo la liberalización del sector cafetalero. Actualmente no existen organismos internacionales de países productores que tengan por objetivo la regulación de la oferta. Los precios del café se determinan en la bolsa de valores y son un ejemplo contundente de las políticas de liberalización económica.

Para México, el café sigue siendo el principal cultivo generador de divisas. No obstante, en las últimas tres décadas su producción y productividad se han visto fuertemente afectadas por el retraimiento de las instituciones del Estado que regularon el sector - Instituto Mexicano del Café, INMECAFETal situación ha podido ser sobrellevada gracias a la sólida capacidad organizativa que expresaron las organizaciones campesinas, en particular, en las zonas mayoritariamente indígenas. Algunos datos muestran enseguida la relevancia del sector y del porqué habrá de encontrar eco en la construcción del Comercio Justo. La liberalización de la cafeticultura sirvió para consolidar un número importante de organizaciones campesinas. En esto, el CJ tuvo un papel preponderante, ya que dio las condiciones para facilitar un proceso de aprendizaje del intrincado proceso de exportación. Actualmente, las organizaciones pioneras del Comercio Justo tienen una sólida postura en su interlocución frente al Estado (Murray, et al., 2003). Sin embargo, el grueso de los pequeños productores de café en México —como en 
el resto del mundo- quedan fuera de los procesos asociativos, lo cual les hace más vulnerables a las crisis económicas.

En México existen más de 400,000 productores, de los cuales $90 \%$ son pequeños, con extensiones de tierra menores a las 5 hectáreas, y 30\% de éstos son mujeres. Es la base económica de 3,200,000 de personas. Se cultiva en las zonas montañosas y se estima que cerca de 60\% de los pequeños productores son indígenas. La producción del país está concentrada en los estados de Chiapas, Puebla, Veracruz y Oaxaca, que representan en conjunto $91 \%$ de la producción, $82 \%$ de la superficie y $80 \%$ del número de productores. De la producción total, $84 \%$ es grano arábigo procesado con beneficio húmedo, 12\% arábigo natural — secado al sol - y el restante $4 \%$ corresponde a la variedad robusta (SAGARPA, 2010).

La permanente baja de precios del café a nivel mundial se hace sentir con igual medida para el grueso de los cafetaleros - pertenezcan o no a las redes de CJ- . La evidencia más dramática de tal hecho es el incremento de los flujos migratorios hacia Estados Unidos en las zonas cafetaleras de México y Centroamérica. Estos efectos de los bajos precios internacionales del café se expresan en igual nefasta magnitud en el resto de los países productores (Raynolds, 2006). Para el caso mexicano, este cultivo sigue ocupando el primer lugar en generación de divisas en el sector agrícola; sin embargo, actualmente el principal rubro en la generación de divisas para el país es el envío de remesas, monto que tiende a equipararse con las divisas generadas por el sector petrolero.

En México, los escasos recursos destinados a la cafeticultura se concentran en aquellos sectores que tienen mayor valor comercial en el mercado internacional. La sólida posición de las organizaciones de CJ como empresas sociales exportadoras les ha permitido negociar ciertos créditos y subsidios a la producción. No obstante, el sector cafetalero -y primario en general - demanda una mayor intervención del Estado que refuerce la producción y productividad, así como una política de precios que dé mayor fortaleza al mercado nacional (Calo y Wise, 2005).

\section{De la aparición del sello Max Havelaar a sus polémicas actuales}

La introducción del sello Max Havelaar ha actuado como elemento distintivo de los productos en el mercado. Todo comienza por la identificación del logo por parte del consumidor y su decisión de adquirir el producto que lo ostenta. Puede pensarse que la medida estuvo orientada a reforzar la construcción identitaria de los consumidores y demás agentes que intervienen en el dispositivo de coordinación. Lo cierto es que también ha sido una eficaz herramienta en la administración financiera de los agentes y ha facilitado la estrategia de márquetin apoyada en la identificación de un logo (Klein, 2001). He aquí, probablemente, uno de los primeros conflictos a los que se ha enfrentado el Comercio Justo. En principio, porque significó aceptar que la buena voluntad de los consumidores no es suficiente para lograr un impacto en el desarrollo de los pequeños productores. Por otro lado, CJ acepta el mercado como mecanismo promotor del desarrollo local, pero considera necesario la definición de reglas que permitan manejar las condiciones desventajosas en que intervienen los pequeños productores del sur.

Los productos pioneros del CJ —café, té, miel, banano y jugo de naranja- son típicamente materia prima importada para su procesamiento y empaque final en los países consumidores. En un primer tiempo, la puesta en operación del nicho de mercado requirió concertar acuerdos con los agentes en las cadenas comerciales de los países consumidores. Un resultado primordial fue consolidar el trato directo entre los pequeños distribuidores del norte con proveedores del sur. En el caso del café, se trata de un producto que adquiere especificidad por la utilización de procesos artesanales en su torrefacción. A este conocimiento artesanal se incorporaron los valores de solidaridad 
movilizados por el CJ, generando una sinergia positiva que se expresó directamente en el incremento del margen de ganancia ${ }^{3}$ del producto. Tal hecho dio un respiro inmediato a los pequeños torrefactores en los países típicamente consumidores, quienes lograron por este medio mantener su nivel de competitividad económica frente a la apabullante competencia de las grandes transnacionales del café.

Ciertamente la relación norte-sur del nicho de mercado de CJ se hizo atractiva para los pequeños importadores, y aquí cabe recordar que esto sucede en pleno auge de la aplicación de medidas de liberalización del mercado. Las grandes compañías - sin importar el tipo de actividad - avasallan con su estrategia de gran escala. Así que una buena opción para los pequeños torrefactores - en el caso del café- fue establecer estas alianzas comerciales con sus proveedores del sur para con ello poder entrar en la lógica de la diferenciación.

Ante la perspectiva del trato directo importadorproductor, se requerían organizaciones campesinas que evolucionaran hacia el concepto de empresas sociales. Esto constituiría la base para romper con la intervención de los intermediarios locales. Fue así que la eliminación de "los coyotes" se convirtió en una de las metas del CJ. El planteamiento es por demás atractivo, ya que permitiría -en teoría - incrementar el margen de ganancia de las organizaciones campesinas y, por ende, de los propios campesinos. Hasta aquí, la propuesta resulta lógica. No obstante, vale la pena recordar - y en especial para el sector agropecuario - que los intermediarios locales en los países del sur cumplen diversas funciones que definen el tejido social de los territorios poco atendidos en términos de servicios públicos.

Yendo un poco más lejos, y haciendo una evaluación de una filière, tendría que señalarse que la introducción del sistema de manejo del sello no modificó las intrincadas transacciones comerciales de intermediación que se dan en Europa, con materias primas como el café o el cacao. ${ }^{4}$ Este aspecto es poco remarcado como debilidad del CJ, argumentando que la originalidad — calidad - de los productos finales depende de la mezcla de diferentes propiedades de las materias primas. Por otro lado, el incremento del margen de ganancia en el dispositivo de CJ no necesariamente se distribuye de manera equitativa entre los agentes que intervienen. Punto que plantea otra de las grandes polémicas que existen en torno al CJ. ¿Quiénes se apropian de ese mayor margen de ganancia? Sin duda, ésta es una pregunta que debería retomarse en la perspectiva de la redefinición de reglas que rigen el CJ.

Hasta ahora hemos mencionado productos agrícolas pero al parecer la dependencia norte-sur se repite en otro tipo de productos. Ciertamente, la comercialización de productos artesanales, tales como textiles, cerámica o joyería, han tenido una importante influencia en el mejoramiento de los ingresos económicos para las mujeres sureñas. Sin embargo, la propuesta es limitada debido a que la aceptación de un producto artesanal depende de patrones de moda ${ }^{5}$ de las sociedades de consumo de los países del Norte (Le Velly, 2006:234; Yépez, et al., 2006). Probablemente, la incorporación de los artesanos del sur al mercado implica no sólo mejorar la calidad en términos de definición del producto, sino también una modificación o pérdida del significado, las representaciones mentales y la identidad étnica, que han permitido el mantenimiento de un saber hacer.

\section{El uso del sello}

Intentaremos seguir el hilo conductor en torno a las implicaciones de la introducción del sello. ¿Qué cambios operativos implicó su introducción? Probablemente al momento de la introducción del sello de CJ no se planteó la necesidad de hacer el control estricto de los agentes que iban a intervenir. La medida pretendía facilitar la identificación del producto para el consumidor. Sería también una manera de generar ingresos para las ONG que promovían la iniciativa, ya que el uso del sello comprometía a un pago por derecho de uso de los 
importadores, procesadores y distribuidores. ${ }^{6}$ Puede considerarse que la operación del sistema estaba basada en relaciones de confianza (Doppler y González, 2006). El número de agentes que participaban en el CJ era lo suficientemente reducido como para permitir que se conocieran unos y otros. Por ende, no hubo incremento en los costos administrativos.

Podría decirse que el mismo éxito alcanzado por CJ trajo consigo la necesidad de agilizar el sistema de control que permitía el uso del sello, medida que permitiría igualmente incrementar los volúmenes de productos comercializados. Fue así como se inician los pasos hacia la certificación del producto con el sello de CJ en un acelerado proceso de institucionalización y profesionalización. Han sido las instancias no gubernamentales quienes han dirigido este proceso desde aproximadamente hace 40 años, habiendo logrado abrirse camino tanto en la aceptación de consumidores reflexivos, como también en el reconocimiento de la propuesta por agencias internacionales de desarrollo y los mismos Estados nacionales.

La definición de criterios evolucionó en función de las relaciones con pequeños productores. Esta etapa del CJ se caracteriza por un esfuerzo permanente por comprender las condiciones de los productores del sur. El objetivo principal fue construir relaciones de proximidad. Objetivo muchas veces compartido por movimientos sociales próximos a $\mathrm{CJ}$ como la agricultura orgánica, la silvicultura sustentable — sellos verdes-, el reconocimiento a los productos locales o produits de terroir. Igualmente, se trata de movilizaciones sociales orientadas a promover el desarrollo local, que en su origen rechazaron las disposiciones impuestas por los modelos intensivos de producción. En todo caso, se trata de la apropiación de un recurso colectivo que remite a sentimientos, valores y representaciones compartidas, materializadas en el cumplimiento de criterios e itinerarios técnicos. Resultado de ello son productos diferenciados, que en función de una calidad específica adquieren un sobreprecio en el mercado.
Finalmente, la problemática que enfrenta CJ no es en modo alguno distante de la que acontece en estos otros dispositivos de comercialización. La certificación se plantea como una solución a problemas de calidad, de aceptación en el mercado de exportación. Teóricamente, las reglas y procedimientos para construir un proceso de certificación es un espacio de negociación de los agentes que intervienen. En el contexto actual, toma mayor importancia el factor de la certidumbre o confianza en la certificación y eficiencia económica. La certificación está actuando como mecanismo para consolidar el posicionamiento de un producto en el mercado. En aras de tales intereses se implanta una regulación de acceso, dictada desde instituciones de los países del norte. El asunto resulta bastante cuestionable, cuando se cierra la participación de aquellos agentes - los pequeños productores de los países del sur-, de quienes se esperaba alcanzaran un mayor desarrollo y autonomía en la toma de decisiones relacionada con la apropiación del recurso colectivo movilizado.

La intervención de FLO, como instancia reguladora para la definición de criterios y operatividad de los valores éticos, no es simple. A nuestro juicio, FLO ha optado por una solución flexible y amplia, que da cabida a varias opciones simultáneamente, siempre en el marco de las definiciones de la Organización Mundial de Comercio (OMC). De esto se deriva el hecho de que el CJ pueda incluir tanto a organizaciones campesinas como a grandes plantaciones. El inconveniente inmediato de esta solución es que el planteamiento deja bastante de lado todo lo que remite a participación de los productores, adhesión a los valores y búsqueda de alternativas de desarrollo.

Una de las vías de solución que se abre con bastante expectativa es la participación gubernamental como instancia reguladora. Veamos hasta qué punto este planteamiento puede representar cierta ventaja. Vale la pena enfatizar que esta voluntad de remitirse al Estado como ente regulador está pensada desde los países del Norte. Tal situación introduce la falta de 
reconocimiento a los procesos locales en los países de Sur orientados en este sentido. El caso específico más consolidado es la articulación de Comercio Justo México, como instancia certificadora en este país y miembro de FLO (Desjardins y Tremblay, 2006).

Los nuevos trabajos con posturas más críticas al CJ aparecen al momento en que se acentúa el incremento de las cifras del negocio de ventas, en detrimento de los procesos de desarrollo y autonomía de los pequeños productores. Las arbitrariedades en la distribución del margen de ganancia para el caso del CJ de café (Pelupessy, 1994); el planteamiento de un proceso de exclusión social frente a la necesidad de regulación de la relación oferta demanda (González, Moguel y Linck, 2002); y la banalización del CJ como concepto de moda (Jacquiau, 2006).

El CJ plantea una relación comercial Norte-Sur, lo que inherentemente implica un mercado internacional; ámbito que tiende a acentuar procedimientos de control más estrictos remitiendo a diversos criterios de calidad. De hecho, la certificación de CJ permite validar la calificación de los valores éticos incorporados y actúa al mismo nivel que cualquier otro proceso de certificación de productos en el mercado internacional. Las reglas para la obtención de la certificación se definen fuera de los espacios locales de los pequeños productores. Esto provoca que tienda a proliferar una percepción bastante amarga del CJ, en el sentido de ser un conjunto de requisitos para poder exportar y no necesariamente una opción para la construcción de procesos de desarrollo local (Doppler y González, 2006).

\section{De las alternativas}

Desafortunadamente, la debilidad del CJ como propuesta de desarrollo local no es un fenómeno aislado. En particular, el movimiento de agricultura orgánica, -fuertemente ligado a la evolución de la cafeticultura indígena que participa en $\mathrm{CJ}$ - ha sufrido desestabilizaciones similares por cuanto a la imposición de reglas emitidas por las agencias certificadoras (González y Nigh, 2005). Los procesos de certificación de CJ y la agricultura orgánica se entrelazan en la práctica. No obstante tal proximidad, no se ha logrado proponer un solo proceso de inspección-certificación. En ambos casos, los costos de inspección y certificación son cubiertos directamente por las organizaciones de pequeños productores. Las organizaciones campesinas de CJ están obligadas a presentar la certificación orgánica, de un sello u otro, en función de los intereses comerciales de los importadores.

La complejidad de los sistemas de control internos y el costo mismo de la inspección de las agencias certificadora elevan directamente los costos de producción. Con el fin de disminuir el peso administrativo de las inspecciones para la certificación, las organizaciones de pequeños productores cafetaleros desarrollaron un eficiente sistema de control interno. Esto se concreta con una serie de inspecciones internas por parte de los técnicos comunitarios de las organizaciones campesinas, quienes llevan un estricto control documental por parcela de cada productor. Para el caso mexicano, los cafeticultores indígenas pueden tener entre 1 hasta 5 parcelas, de 0.5 y 5 ha, distribuidas en más de una localidad. Esta distribución es el resultado de complicados procesos de reestructuración agraria, pero también ha servido para asegurar una mayor diversidad de condiciones microambientales. Las consecuencias son positivas en términos de reducción de riesgos por fenómenos climatológicos; sin embargo, representa una dificultad por lo que concierne a los tiempos de recorrido entre las parcelas que, por ende, eleva los costos. Estos técnicos comunitarios son los responsables de dar la asesoría técnica y organizar cursos específicos para el fortalecimiento de las reglas técnicas de la agricultura orgánica y de los postulados del comercio justo.

Los procesos de innovación de las organizaciones campesinas, en colaboración con FLO, han consolidado la puesta en marcha de un programa piloto con 
cooperativas de café en el sur de México, ${ }^{7}$ mismo que pretende establecer una sola inspección para obtener las certificaciones de CJ y de agricultura orgánica. Estas son iniciativas concretas cuyos resultados positivos podrían fortalecer dinámicas locales, sin descuidar los aspectos de confianza para el consumidor.

Los ejemplos que han sido abordados muestran el caso de productos que al ser certificados permiten el acceso a un sobreprecio o cuotas sociales. Caso distinto se presenta para las certificaciones de silvicultura comunitaria sustentable, ${ }^{8}$ ya que la obtención del certificado no garantiza un sobreprecio. Sin embargo, su operación en países del sur ha sido adoptada con gran participación por organizaciones campesinas comunitarias. Ciertamente, las organizaciones campesinas han accedido a este tipo de certificaciones con el apoyo financiero de diversas fuentes no gubernamentales y gubernamentales; no obstante, los retos que deben enfrentar son nuevamente la competencia por mayor eficiencia económica frente a las grandes compañías del sector forestal (Taylor, 2005).

Unúltimo caso que deseamos utilizar como ejemplo de los procesos de apropiación de los recursos colectivos son las Apelaciones de Origen Controlado (AOC). En modo alguno estamos saliendo del ámbito de discusión, ya que el planteamiento sigue siendo válido, en la medida que el caso envía a la movilización de un recurso colectivo - territorio - a través del mercado. Las opciones de diferenciación territorial de los pequeños productores son igualmente una vía para el acceso a un sobreprecio en un mercado de nicho. En esta forma, el planteamiento es el mismo que el seguido por CJ. La AOC remite al concepto de Indicaciones geográficas (GIS) "which identify a good as originating in the territory of a member country, or a region or locality in that territory, where a given quality, reputation or other characteristics of the good is essentially attributable to its geographical origin" (Barham, 2003: 127). Esto mismo cobra operatividad comercial en el marco del General Agreement on Tariffs and Trade (GATT), específicamente en lo que corresponde a lo enunciado en el Agreement on Trade-Related Aspects of Intellectual Property Rights (TRIPS), marco jurídico internacional para los acuerdos comerciales. La definición del origen territorial remite a características que se definen por las condiciones geográficas, pero también para los saberes locales puestos en juego para producir determinado alimento o bebida. En principio, esto podría ser una ventaja para los pequeños productores y para la promoción del desarrollo rural; sin embargo, plantea igualmente una exclusión ya que sólo se benefician los productores que se inscriben en el territorio del GIS (Barham, 2003; OMC, 2006).

Hasta aquí el planteamiento parece positivo. Aunque existen fuertes deficiencias en los acuerdos comerciales que están generando situaciones desventajosas en el sector agroalimentario para los pequeños productores. En primer lugar, el procedimiento para obtener el registro de un indicador geográfico demanda una gran inversión de tiempo y capital —en el sentido amplioque no siempre es accesible para un grupo de pequeños productores. Derivado de ello, frecuentemente se realizan alianzas "estratégicas" con el fin de permitir una entrada más amplia o menos rígida de lo que originalmente se había definido en la calidad geográfica, dando cabida al término free riders o "gorrón" —en el caló mexicano-. Es por ello que en este trabajo pretendemos remarcar no sólo las contingencias que enfrenta CJ, sino de igual manera las vicisitudes de los pequeños productores frente al marco jurídico del mercado internacional.

\section{Introducción de una política de Estado para la regulación del mercado}

Actualmente, en algunos países europeos existe una presión de diversos grupos sociales para exigir a sus gobiernos acciones concretas que beneficien al Comercio Justo. Lo motivos podrían ser agrupados en dos grandes paquetes: 
1. Exigencia ciudadana a los Estados nacionales en los países del norte, para tomar acciones concretas que redunden en el desarrollo sustentable y en relaciones que ponderen la justicia social con los países del sur.

2. Preocupación del Estado por proteger a los consumidores y, en general, por crear un marco jurídico institucional que brinde certidumbre a los agentes comerciales que intervienen en el Comercio Justo.

Estas preocupaciones de los gobiernos en países del norte surgen del debate de una teoría económica neoinstitucionalista, misma que considera la relación Estado y mercado más allá de una postura de confrontación, y que con la participación de la sociedad civil promueve la construcción de los procesos sociales (Valdivieso, 2006). En esta medida, la sociedad civil deviene como agente transformador, tal como lo ha demostrado la participación de Organismos No Gubernamentales (ONG) en la elaboración de marcos normativos. En este orden se ubica el movimiento de agricultura orgánica emprendido por la International Federation of Organic Agriculture Movements (IFOAM). Remitimos a esto porque es sin duda un claro ejemplo de un movimiento emprendido por la sociedad civil estrechamente ligado al CJ, cuyo posicionamiento a escala mundial condujo a la intervención del gobierno. El respeto de los criterios ambientales de conservación está asociado con la salud del consumidor y con la conciencia de éste, al reconocer la necesidad de pagar un sobreprecio a los productores. La intervención del Estado en el sector de la agricultura orgánica podría dar pauta a ciertas consideraciones para procesos similares que involucren a pequeños productores en países del sur.

Esevidentelainfluenciadelateoríaneoinstitucionalista en la propuesta de CJ. El planteamiento de North (1991) enfatiza el rol de las instituciones como "restricciones ideadas por los humanos que permiten estructurar los intercambios económicos, sociales y políticos" (North, 199l: 3, citado por Valdivieso, 2006). Las reglas formales se plantean como formas de regulación, que cobran cuerpo en la elaboración de leyes o en los derechos de propiedad, donde el Estado tiene un papel preponderante. Por otra parte, las reglas informales se basan en dispositivos de coordinación, que se concretan en "códigos de conducta autoimpuestos", tales como estándares de honestidad e integridad y normas de comportamiento que se refuerzan por la sanción social o la autoridad coercitiva. Los mecanismos que permiten regular o imponer tales normas también pueden ser considerados instituciones $\mathrm{y}$, de hecho, juegan un papel significativo en este enfoque, ya que:

...el grado de identidad entre los objetivos de una pauta institucional y las elecciones individuales depende de la efectividad de los mecanismos de refuerzo, los cuales pueden provenir de una interiorización de las normas, de la sanción social o imposiciones coercitivas del Estado (North, 1990: 6, citado por Valdivieso, 2006).

Por otro lado, en esta perspectiva las reglas informales constituyen un conjunto fundamental de instituciones consistentes en convenciones comunes destinadas a resolver problemas de coordinación, que pueden ser, alternativamente:

...códigos de conducta autoimpuestos, tales como estándares de honestidad e integridad, y normas de comportamiento que se refuerzan por la sanción social o la autoridad coercitiva (Valdivieso, 2006: 25).

Siguiendo el planteamiento de North, se entiende que la redistribución de la riqueza se basa en la habilidad institucional para asegurar los derechos de propiedad y los contratos.

Si bien el planteamiento de North resulta válido, vale la pena preguntarse si las instituciones en los 
diferentes ámbitos territoriales (regionales, nacionales e internacionales) son lo suficientemente sólidas para cumplir el rol que se les asigna. Expresado en otras palabras, ihasta qué punto las instituciones están permitiendo espacios democráticos que aseguren la vigilancia en la construcción de marcos jurídicos, su acatamiento y la aplicación de sanciones? En este sentido, señalamos la necesidad de hacer una reconsideración sobre la participación ciudadana en las instituciones que coordinan y regulan el Comercio Justo.

\section{¿Qué dicen los estudios ex profeso para la toma de decisiones?}

El estudio preparado por Cora Dankers para la FAO (2004) es probablemente uno de los más completos sobre CJ y temas relacionados. La autora explora el origen de estas iniciativas, sus alcances y sistemas de certificación. Asimismo analiza la participación de los interesados, el proceso para el establecimiento de normas, los métodos de verificación, la relación con los Acuerdos de OMC y el posible papel de los gobiernos. Veintidós estudios de casos ilustran el impacto que las normas y programas de certificación han tenido sobre los costos de producción y los ingresos de los productores en los países en desarrollo, con datos sobre los mercados del banano, café, té y cítricos, lo que resulta herramienta importante para la toma de decisiones de política pública (FAO, 2004).

Este trabajo parte de un mosaico de casos que articula procesos sociales de ámbito local con la especificidad de los marcos jurídicos de organismos internacionales. Reconoce la restricción de las propuestas de ONG, gobiernos e iniciativa privada en el marco de los acuerdos de la Organización Mundial del Comercio (OMC). Particularmente, se refiere al principio no discriminatorio de productos similares, revisando las definiciones de la Nación Más Favorecida (NMF) y Trato Nacional (TN). La OMC actúa en todo momento como la garante de la libre competencia; reconoce y acepta la firma de acuerdos voluntarios entre agentes de mercado pero sanciona cualquier condición que brinde ventaja a alguno de los participantes. Esta condición, expresada de manera bastante simple, es "la camisa de fuerza" a la que está sometido el CJ, o cualquier otra iniciativa que tenga como objetivo ofrecer mejores condiciones a los productos provenientes de países del sur.

Dankers señala igualmente la falta de regulación internacional para ejercer un mayor control sobre el acatamiento de los derechos laborales en los países del sur. La adhesión a los principios de la Organización Internacional del Trabajo (OIT) constituye un punto neurálgico como estrategia de desarrollo, aunque la observación de estas reglas no se considera competencia de OMC. Paralelamente, los gobiernos del sur se caracterizan por su debilidad y para hacer cumplir los derechos laborales de sus ciudadanos.

Con el afán de atenuar la desventaja de los trabajadores del sur, han surgido diversas iniciativas que pregonan el respeto a los derechos laborales. Las formas para ratificar este compromiso se llevan a cabo por dos vías. Una de ellas es la adhesión a códigos de conducta voluntarios definidos por las mismas empresas. La desventaja de este tipo de sistema es la participación como juez y parte y, por ende, su baja credibilidad. Por ello, la certificación por un tercero ha devenido como la vía más recurrente.

En consecuencia con los derechos laborales internacionales, el CJ tiene criterios que permiten evaluar su cumplimiento. Ciertamente, esta consideración es válida únicamente para el caso de productos provenientes de empresas privadas (plantaciones o talleres artesanales). Se promueve la vida asociativa de los trabajadores como un mecanismo que permite posibilidades de desarrollo. Sin embargo, el respeto a los derechos de trabajadores agrícolas eventuales es un aspecto que ha sido bastante olvidado por el CJ. Tanto en las grandes plantaciones como en las unidades de pequeños productores, que manejan cultivos como 
banano, té o café, se requiere la mano de obra externa en determinados momentos del ciclo productivo donde la mano de obra familiar no es suficiente. Un ejemplo típico de esta situación es durante la cosecha de café. Ciertamente, si esto se tradujera en un criterio de CJ los pequeños productores incrementarían sus costos de producción de mano de obra, sin precisamente generarles un mayor ingreso. De ahí que siga imperando el anonimato de los trabajadores eventuales.

El vacío institucional gubernamental, en ámbitos como los derechos laborales o el control sobre políticas de conservación y manejo de recursos naturales, deja paso a la intervención de iniciativas privadas o de ONG. No obstante los aspectos positivos que cada uno de estos sellos puedan representar, es imperioso notar que sus alcances distan mucho de resolver el problema de fondo. Se trata, en todo caso, de aspectos donde sin duda se reclama la intervención gubernamental (Gendron, 2006).

Un segundo informe al que haremos referencia es el presentado por la Cooperación Técnica Belga (СТВ, 2006). Este documento se encuentra orientado en gran medida a la discusión del gobierno belga respecto a la propuesta de ley referente al CJ. El objetivo es alcanzar un nivel supranacional en la Unión Europea. La CTB realizó un análisis de Fuerzas y debilidades, oportunidades y amenazas (FODA) de diversas iniciativas de CJ emprendidas por ONG y gubernamentales. Éste señala la influencia que dicho tipo de iniciativas de ley han tenido en función del juego de los partidos políticos que las enarbolan -caso específico de Francia-. Recalca igualmente la debilidad de los gobiernos para ejercer su facultad como entes reguladores de los agentes del mercado - con particular referencia al sello social del gobierno belga- Coincide con el estudio de Dankers (2003) en señalar que el éxito de una propuesta de ley debe conciliar evidentemente los agentes que intervienen. Remarca igualmente las constricciones en cuanto a los acuerdos internacionales que refiere la OMC. Más allá de la postura señalada en el citado informe, la Cooperación Técnica Belga (СТВ) anuncia su contribución a un fondo de capital que sirva como aseguramiento a las compañías importadoras (Fair Trade Center Belgique, 2009). Esta medida pretende coadyuvar a las limitaciones que existen para hacer un pago anticipado a los productores del sur. Más allá del debate político, este tipo de acciones muestra el reconocimiento de los problemas de fondo y la voluntad de colaborar en soluciones viables. Sin embargo, alcanzar este objetivo sigue siendo un problema de disponibilidad de fondos financieros. ${ }^{9}$

\section{El escenario en Bélgica}

Unalecturadelasúltimas acciones en tornoal CJ muestran la participación de CTB como promotora de espacios públicos de discusión. En octubre de 2006 se realió un foro abierto, donde fueron invitados representantes de las iniciativas pioneras de CJ: Oxfam (Wereldwinkel y Magasins du Monde) y Max Havelaar; compartiendo el foro con versiones más recientes de mercados solidarios, específicamente: Rainforest Alliance, Utz Kapeh (código de conducta EUREPGAP) y el proyecto $4 \mathrm{C}$ (Codes d'usages Communs pour la Communauté du Café) (Fair Trade Center Belgique, 2006).

El foro evidenció el poder de convocatoria de CTB frente a las agencias de CJ en Bélgica; sin embargo, dejó igualmente claro que las diferentes iniciativas no están preparadas y mucho menos interesadas en la unificación de estándares y procedimientos. Aceptan que cada propuesta tiene objetivos y procedimientos que tienden a posicionarse en segmentos de demanda que no compiten entre sí. Para el caso particular de Max Havelaar-Belgique, la posiblidad de crear estándares comunes corre el riesgo de crear lo que ellos llaman "la versión light du Commerce Équitable", ya que implicaría hacer disponible un producto que tratando de ser competitivo en el mercado ofreciera un precio atractivo para el consumidor, en menoscabo del precio pagado al productor. 
Las diferentes iniciativas dieron información respecto a sus estrategias de financiamiento. En algunas de ellas, los fondos gubernamentales juegan un papel importante en el cumplimiento de sus funciones. Este hecho muestra los distintos grados de compromiso de los Estados nacionales en los países consumidores. Destaca también la postura de aquellas iniciativas de comercio solidario que se mantienen en la esfera de lo privado, en lo que probablemente remite a la ética empresarial utilizada comúnmente como estrategia de mercadeo (EUREPGAP).

La definición de criterios comunes está lejos de ser posible. En consecuencia, los niveles de participación de los pequeños productores se mantienen como un punto bastante vulnerable. Si bien la mayoría de las iniciativas aseguran que parten de una relación de confianza con los productores, en el foro antes citado el representante de Oxfam reconoció que "los productores no siempre nos tienen confianza. El comercio justo sigue siendo por el instante un asunto del Norte con las normas éticas del Norte aplicadas al Sur. Nosotros reflexionamos demasiado con un razonamiento del Norte" (Fair Trade Center, Belgique, 2006). ${ }^{10}$ He aquí uno de los puntos débiles que desde la perspectiva del sur requiere una urgente reformulación (Doppler y González, 2006a, y 2006b). Cuestiones que siguen en permanente discusión son, por ejemplo, la pertinencia y métodos para fijación del precio mínimo o la propuesta que plantea favorecer a través del CJ a regiones geográficas con necesidades socioeconómicas más agudas, como el caso de África sobre Latinoamérica, o la conciencia ciudadana en el norte que tiende a promover un mercado solidario que beneficie a los pequeños productores de los mismos países del norte.

\section{Consideraciones finales}

Al parecer existe una voluntad política para definir un marco jurídico supranacional de CJ. Es un hecho que habrá de concretarse tarde o temprano. Los primeros pasos se han dado. Lo importante será no perder de vista los objetivos que privilegian el desarrollo local de los pequeños productores. Habrá que considerar que la economía - como disciplina - no tiene elementos teóricos que permitan discutir la fijación de los precios con la incorporación de los valores éticos. Sus planteamientos remiten a lógicas de competitividad que relacionan básicamente oferta y demanda. Siendo así, nos queda claro que estamos en un ámbito no escrito. ¿Será posible fijar nuevos procedimientos para la fijación de precios? ¿Cómo resolver las contradicciones que plantea un mundo global de libre mercado frente al reconocimiento de las desventajas de los países menos desarrollados? Hasta ahora, los esfuerzos para generar tal marco jurídico están centrados en generar un proceso ágil y confiable, prevaleciendo el sentido de armonizar los estándares del Comercio Justo. Sin embargo, nosotros enfatizamos la desventaja que tal proceso puede plantear. Las experiencias de armonización en diversos ámbitos de la producción, ligados al comercio internacional, dan muestra de sus limitaciones; sobre todo, mientras haya un marco comercial restrictivo. Es decir, mientras las reglas de GATT no sean replanteadas, no queda gran margen de maniobra. Resultado de ello serán criterios que tiendan a definir criterios de solidaridad bastante laxos, que seguirán marcando como condición la libre participación de los agentes comerciales y, por ende, los pequeños productores del sur seguirán sin posibilidades de ser los beneficiarios específicos.

Por otro lado, es un hecho igualmente innegable que armonizar el CJ representa un desafío bastante complicado. Tratar de enlazar la lógica del los productores del sur con la de los consumidores del norte resulta bastante atrevido. Más allá de esto, la evolución de $C J$ en los países consumidores tiene procesos extremadamente distintos. No será a través de una regla que logremos estandarizar las dinámicas y volúmenes de ventas de CJ. Habría que reconocer que ante todo CJ es una forma de expresar nuestros sentimientos y nuestras 
expectativas de justicia social. El reto está no sólo en plantearse un mercado que favorezca a los pequeños productores del sur, sino también en establecer nuevas formas de producción y criterios para la definición de los precios. Mientras domine el criterio de eficiencia y rentabilidad económica estaremos favoreciendo formas intensivas de producción. De esto ya conocemos las consecuencias y las facturas nos están llegando por daños ambientales y desigualdad social. No puede pensarse que un mercado de nicho logre resolver las desigualdades tan abismales existentes en la sociedad global.

Las acciones de CJ han privilegiado los criterios que apuntan a dar confianza en la cadena productiva que certifican — sea café, té, banano, cacao, o cualquier otro-, productos básicamente orientados a la exportación, pero han descuidado los criterios que podrían activar procesos de desarrollo local; en especial, las acciones de diversificación productiva, el fortalecimiento de los mercados locales del sur y, sobre todo, procesos educativos que permitan el arraigo de las nuevas generaciones a sus territorios. ¿Qué se requiere para lograr esto? En un primer paso, dar mayor participación a las organizaciones campesinas en la definición de las reglas del CJ, sabiendo que sobre todo esto implicará un esfuerzo especial para entrar a dos mundos cognitivos extremadamente distintos: el de los hombres que dominan el espacio de los intercambios comerciales y fijan las reglas escritas, y el de los hombres y mujeres campesinos que construyen su conocimiento a partir de la oralidad.

\section{Notas}

${ }^{1}$ Este documento es uno de los resultados de la estancia postdoctoral con una beca de la Université Catholique de Louvain, en Bélgica, durante el periodo julio 2006-agosto 2007.

${ }^{2}$ Incluye a SAN (Red de Agricultura Sostenible), FLO (Organización de Etiquetado de Comercio Equitativo),
IFOAM (Federación Internacional de los Movimientos de Agricultura Biológica), IOAS (Servicio Internacional de Acreditación de la Producción Orgánica) y SAI (Social Accountability International).

3 Incrementar el margen de ganancia no implica automáticamente que todos los agentes se benefician por igual (Pelupessy, 1994).

${ }^{4}$ El manejo de diversos orígenes de la materia prima sirve como ejemplo muy claro para el caso del café y el té. Sin embargo, el argumento puede repetirse para muchos otros productos orientados a la exportación en los países del sur. El mismo argumento resulta válido para productos como cacao (contenido de flavonoides) y algodón (resistencia, color y largo de fibras) -tan sólo por hacer un simple ejercicio de la validez de la argumentación.

${ }^{5}$ Esta misma situación se presenta en el caso de flores, sector extremadamente ligado a los lineamientos de la moda que fijan los diseñadores de arreglos florales en los países del norte.

${ }^{6}$ Para mayor información sobre la fase pionera de la iniciativa Max Havelaar remitirse a la obra de sus fundadores (Rozen, N. y F. van der Hoff, 2002).

${ }^{7}$ Existen algunos esfuerzos en este sentido pero se limitan a programas piloto, algunos de ellos ubicados en Oaxaca y Chiapas.

8 Proyectos financiados por Fair Trade and Forest Stewardship Council (FSC) (2009). Hace una breve presentación sobre experiencias de silvicultura sustentable en el estado de Oaxaca, México.

${ }^{9}$ Entrevista realizada a colaborador del Fair Trade Cente de la Cooperation Technique Belge, [noviembre de 2006].

${ }^{10}$ Traducción propia.

\section{Bibliografía}

Barham, E. (2003), "Traslating terroir: the global challaenge of French AOC labeling", en Journal of Rural Studies 19, pp. 127-138. 
Bhagwati, J. y R. E. Hudec (Eds.) (1996). Fair Trade and Harmonization. Prerequisites for Free Trade?, vol. 2, Massachusets, USA: The MIT Press.

Calo, M. y Timothy A. Wise (2005), Revaluing Peasant Coffee Production: Organic and Fair Trade Markets in Mexico. Global Development and Environment Institute, Tufts University. http://www.boncafe. org/Documentos/ESTUDIOSBC/12/Organic\%20 Fairtrade\%20Coffee\%20Mexico\%2005.pdf [agosto 2009].

Coopération Techinique Belge (2006), Le commerce équitable en Belgique: Quelles pistes de reconnaissance officielle? Reporte final presentado por: Marie d'Huart, Gerda Heyde y Serge De Backer. www.befair.be/site/ download.cfm?SAVE=1060e LG=3, [junio 2009].

Desjardins, M.C. y A. Tremblay (2006), "Reflexions sur un encadrement juridique des produits équitables au Canada", en ponencia del 2do Colloquio Internacional sobre el Comercio Justo. Montreal, Québec, Canadá: Chaire de responsabilité sociale et de développement durable ESG-UQAM.

Doppler F. y A. A. González (2006a), "Los sabores y sinsabores del Comercio Justo", en Revista Puente @ Europa, año IV, núm. 2, pp. 31-35, http://www.fao.org/ docrep/008/y5136s/y5136s08.htm [agosto 2009].

Doppler F. y A. A. González (2006b), "Comercio Justo: Entre la institucionalización y la confianza”, en Problemas del Desarrollo: Revista Latinoamericana de Economía, vol. 38, núm. 149, pp. 181-202, Universidad Nacional Autónoma de México, y en http://www. ejournal.unam.mx/pde/pdel49/PDE003814909.pdf [agosto 2009].

Fair Trade and Forest Stewardship Council (FSC), http://www.laneta.apc.org/pasos/ffscl.htm, y http://base.d-p-h.info/fr/fiches/premierdph/fichepremierdph-1860.htmlh [junio 2009].

Fair Trade Center, Belgique (2009), "Les objectifs du millenaire pour le developpement", dans http://www. dgdc.be/fr/campagne_omd/actualites.html [junio de 2009].
Fair Trade Center, Belgique (2006), "Le comerce équitble à la croisée des chemins?", dans http://www.befair.be/ fr/articles/www-befair-be/2-ressources/commerceequitable/quelques-etudes-et-rapports.cfm. [junio 2009].

Fair Trade Labelling Organisations (2010), Growing stronger together, Reporte anual 2009-2010, en http://www.fairtrade.at/pics/infomaterial/FLO_ AnnualReport2009.pdf [agosto 2010].

Fair Trade Labelling Organisations (2011), http://www. fairtrade.net/coffee.0.html? \&\&L=1 [febrero de 2011].

Gendron, C. et M.-F. Turcotte (2006), "Mouvements sociaux économiques et gouvernance: une nouvelle structuration du marché?", dans Les cahiers de la Chaire, collection recherché, núm. 14, pp. 20, http://www.crsdd.uqam.ca/Pages/docs/ pdfCahiersRecherche/2006/14-2006.pdf [junio 2009].

Gendron, C., V. Bisaillon y A. I. Otero (2006), "The Institutionalization of Fair Trade: More than a Degraded Form of Social Action", dans Les cahiers de la Chaire-collection recherché, núm. 12, École des sciences de la gestion, Université du Québec à Montreal, http:/www.crsdd.uqam.ca/Pages/docs/ pdfCahiersRecherche/2006/12-2006.pdf [agosto de 2009].

Gómez, L., L. Martin, M. A. Gómez y T. Mutersbaugh (2005), "Certified organic agriculture in Mexico: Market connections and certification practices in large and small producers", in Journal or Rural Studies, núm. 2l, pp. 461-474.

González A. A. y R. Nigh (2005), "Smallholder participation and certification of organica faro in Mexico", in Journal of Rural Studies, núm. 21, pp. 449460.

González, A. A. (1999), Les règles du jeu du marché solidaire. Memoire du DEA-ESSOR (Espaces, sociétés et dynamiques rurales), Francia: ENFA ToulouseAuzeville-ENSA Toulouse-Université Toulouse LeMirail. 
González, A. A. (2002), "Evaluación de los beneficios actuales y el potencial para el combate a la pobreza de la participación en redes de comercio justo de café. Unión de Sociedades de La Selva, Federación de Sociedades de Solidaridad Social", en Fair trade research group-Colorado State University y Desarrollo Alternativo, A. C., http://welcome2. libarts.colostate.edu/centers/cfat/wp-content/ uploads/2009/09/Case-Study-Uni\%C3\%B3n-LaSelva-Chiapas-Mexico.pdf [agosto 2009].

Hernández Castillo, R. A. y R. Nigh (1998), “Global processes and local identity among Mayan coffee growers in Chiapas, Mexico", en American Anthropologist, vol. 100, núm. 1, pp. 36-147.

Jacquiau, Christian (2006), Les coulisses du commerce équitable: mensonges et vérités sur un petit business qui monte, Paris: Mille et une nuits.

Klein, N. (2001), No logo. El poder de las marcas, Barcelona: Paidós.

Le Velly, R. (2006), "Le commerce équitable: des échanges marchands contre et dans le marché", en Revue francaise de sociologie, núm. 47, vol. 2, pp. 319340.

Moguel, P. y V. M. Toledo (1990), "Biodiversity conservation in traditional coffee systems of Mexico", en Conservation Biology, núm. 13, pp. 11-21.

Murray, D., L. T. Raynolds y P. L. Taylor (2003) One cup at a time: poverty, alleviation and fair trade coffe in Latin America, Fort Collins, Colorado State University, Fair trade research group, http://www.usaid.gov/our_work/environment/ compliance/ane/workshops/Jordan2007/day3/S/ FairTradeandPovertyReductionStudy.pdf [marzo de 2011].

Murray, D., L. T. Raynolds y Peter L. Taylor (2006), "The future of Fair Trade coffee: dilemmas facin Latin America's small-scale producers", en Development in Practice, núm. 2, vol. 16, pp. 179-192.

Nico Roozen y Frans van der Hoff (2002), L'aventure du commerce équitable: une alternative à la mondialisation sauvage par les fondateurs de Max Havelaar, Paris: J.C. Lattès.

Organización de las Naciones Unidas para la Agricultura y la Alimentación (2004), Las normas sociales y ambientales, la certificación y el etiquetado de cultivos comerciales, preparado por Cora Dankers, Roma: http://www.fao.org/docrep/008/y5136s/y5136s00. htm [agosto 2009].

Pelupessy, W. (1994), "El mercado solidario de café", en Crisis y perspectivas del café latinoamericano, Comp. Mario Samper K., San José: C.R. UNA-ICAFE, pp. 239-259.

Raynolds, L. T. (2000), "Re-embedding global agriculture: the international organic and fair trade movements", en Agriculture and Human Values, núm 17, pp. 297-309.

SAGARPA (2010), Escenario actual del café. Dirección General de Operaciones Financieras Dirección de Estudios y Análisis de Mercados, http://www. infoaserca.gob.mx/fichas/ficha29-Cafe20100716.pdf [marzo 2011].

Service Public Fédéral, Belgique, Affaires étrangères, Commerce extérieur et Coopération au Développement. Direction générale de la Coopération au Développement (2007), Rapport au Parlement sur l'action de la Belgique en vue de la réalisation des Objectifs du Millénaire pour le Développement (OMD), http://www. dgdc.be/fr/campagne_omd/actualites.html [octubre 2009].

Soto, M.L. (2002), "Shade over coffee: its effects on berry borer, leaf rust and spontaneous herbs in Chiapas, Mexico", en Agroforestry Systems, vol. 55, num. 1, pp. $37-45$

Tallontire, Anne (2001), "Challenges facing Fair Trade: which way now?", en Paper for the DSA conference 2001, Different Poverties, Different Policies, Manchester: IDPM, 10-12 September 2001.

Taylor, L. P. (2005), "In the Market But Not of It: Fair Trade Coffee and Forest Stewardship Council Certification as Market-Based Social 
Change", in World Development, vol. 33, núm. 1, pp. 129-147.

Valdivieso, S. (2006), "¿Nuevos aires de la teoría del desarrollo? Problemas del Desarrollo", en Revista Latinoamericana de Economía, vol. 37, núm. 144, pp. 11-31.
Yépez, I. y M. Mormont (Coords.). S. Charlier, I. Haynes, A. Bach, A. Mayet (2006), Le commerce équitable face aux nouveaux défis commerciaux: évolution des dynamiques d'acteurs, Rapport final, Partie 1, Modes de production e te consommation durables, Paris. 